\title{
THE DETERMINATION OF THE CONSTANT OF A SOLENOID.*
}

\author{
BY \\ S. R. WILLIAMS, PH.D., \\ Professor of Physics, Oberiin College, Oberlin, Ohio.
}

WHETHER measuring the earth's magnetic field by means of an earth inductor or the field in the slit of a radially divided toroid by means of a few turns of wire, the exploring coil has become a useful adjunct in measurements of magnetic field intensities. Particularly is this true since the advent of sensitive moving coil galvanometers. In order to know a magnetic field completely, both its intensity and distribution must be determined. Of the varied and many means at our disposal for thus mapping out a magnetic field, perhaps none lends itself so readily to both field intensity measurements and topographical surveys as does the exploring coil. This adaptability is well illustrated in the following special application:

\section{TESTING THE CONSTANT OF A SOLENOID.}

The constant of a solenoid which has been carefully wound may be found with considerable accuracy from its dimensions, so that experimental tests on the constant may be checked up very closely. Even with the greatest care in winding we are always glad to avail ourselves of any means that will assure us whether any short circuits have occurred during the winding. In other words, we want the satisfaction which comes from contemplating, side by side, the calculated and observed values and finding that they agree.

After winding several solenoids and attempting to verify their constants experimentally by various means, I have found the following method a very practical one. It is based on an old method, common to many labọratories, in which a magnetometer needle is placed at the centre of two coaxial coils, one of which is a standard coil and the other the one to be tested, and

* Communicated by the author. Read by title before the Ohio Academy of Science, Columbus, Ohio, November 28 , 19i4. 
the deflections compared when the fields of the two coils reinforce and when they oppose each other. In the measurement of magnetic fields we may make use of the principle that a magnetometer may be replaced by an exploring coil in circuit with a ballistic galvanometer. We thereby gain all the advantages which a moving coil galvanometer offers over a moving magnet type, chief of which is the freedom from magnetic disturbances. In the above magnetometric method referred to, therefore, we may replace the magnetometer by an exploring coil in circuit with a ballistic galvanometer. In the case of testing a long solenoid, whose inner diameter is very small, the magnetometer is practically out of the question. One solenoid which the author wound was I $00 \mathrm{~cm}$. long and had an inner free diameter of $2.54 \mathrm{~cm}$. Not only did the small inner diameter necessitate a very short sus-

FIG. I.

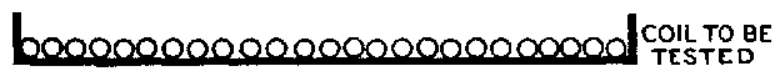

L ITAMDARD COIL

beamad EXPLORING COIL

10800000

p0000000000000009

\section{p000000000000000000000000}

pending fibre for the needle of the magnetometer, but the deflections of the needle could not have been read with any accuracy, since the magnet must necessarily be placed at the centre of the long, narrow opening of the coil.

With the exploring coil replacing the magnetometer (Fig. I), let the two coaxial coils, centred at the same point, be connected in series and the current, $I$, flowing through them so directed that the fields reinforce each other. If $S$ is the constant of the solenoid and $G$ that of the standard coil, then when the current is suddenly broken in the circuit the induced electromotive force in the exploring coil will set up a current through the ballistic galvanometer such that its deflection. $d_{2}$, will be given by the relation :

$$
I S+I G=k d_{3} \ldots \ldots \ldots \ldots \ldots \ldots \ldots \ldots(\mathrm{I})
$$

where $k$ is a constant dependent upon the ballistic galvanometer. 
By reversing the current in one coil and not in the other and again suddenly breaking the circuit, a deflection, $d_{1}$, is obtained. such that

$$
I S-I G=k d_{1} .
$$

where the field of the coil, $G$, has been reversed. Dividing equation (I) by equation ( 2 ) and solving for $S$, we obtain

$$
S=G \frac{d_{2}+d_{1}}{d_{1}-d_{1}}
$$

which is the constant of the solenoid in terms of the constant of the standard coil, and the deflections of the ballistic galvanometer for the two conditions. It will be noted that so long as the current remains constant the current $I$ does not enter into the computation, neither does the constant of the ballistic galvanometer. For measuring the current through the coils all that is needed is an ammeter which will indicate that the current is constant when the fields reinforce and when they oppose each other. When the current is supplied from a storage battery it is easily kept constant enough.

Equation (3) should apply whether the field through the exploring coil is changed by suddenly breaking the electric current or by suddenly rotating the exploring coil through I 80 degrees. For a solenoid with a large inside diameter, the author feels that the rotating exploring coil may be used to advantage, as by its use the solenoid circuit does not need to be disturbed for a series of readings and the integral electromotive force is also double that obtained when the field is simply made zero in the coil. Both the rotating and fixed types of exploring coil were used in testing a coil employed in previous work ${ }^{1}$ and no difference in results greater than experimental errors could be detected.

The question may be raised, Why determine the constant of a solenoid ${ }^{2}$ in terms of another coil whose constant is ordinarily obtained from its dimensions, although not necessarily so? In the experiment on which this work is based, a tangent galvanometer coil was used for the standard coil, and we may answer

${ }^{1}$ Phys. Rev., vol. 34, p. 4o, Jan., 1912.

' $\mathrm{By}$ a solenoid is meant a coil whose constant is computed by the formula,

VoL. 182, No. $1089-26$

$$
H=4 \pi n I \frac{l}{\sqrt{l^{2}+r^{2}}}
$$


the above question by saying that the constant of the tangent galvanometer coil is easily computed and not so much risk arises in the winding that there will be short circuits. Furthermore, if the constant of the solenoid determined in terms of the constant of the tangent galvanometer coil does check with that obtained by computations from the dimensions of the solenoid, we feel a greater confidence that the value of the constant we have found is the correct one. There are, however, objections to the tangent galvanometer coil as a standard. These are: (I) the field at the centre is not uniform enough over a sufficiently large space at its centre and (2) errors in measuring the dimensions creep in too easily. The first objection may be overcome by using a Helmholtz coil, but objection (2) holds even more strongly for the last type of coil. Watson, ${ }^{3}$ in measuring the horizontal component of the earth's magnetic field, used a Helmholtz coil, and, in order to obtain an accuracy of one part in I0,000, used a coil $60 \mathrm{~cm}$. in diameter and measured the radius down to 0.003 of a centimetre. This size of a coil is inconvenient to use as a standard in this work, and a smaller coil decreases the accuracy of the work.

If we examine the formulæ for the constants of various types of coils we find that the one used in computing the constant of a solenoid has fewer quantities which are affected by the dimensions of the coil. The formula is practically dependent only on the number of turns, $n$, per unit length, which, as will be shown later, may be made very accurate. Accordingly it was thought advisable to adopt the solenoid type of coil as a standard, making the diameter of the same as small as practical and placing it inside of the solenoid or coil to be tested. From equation (3) it is evident that so far as theory goes the standard coil may be made coaxial either outside or inside the other coil.

In the work as actually carried out the standard coil was made up in the following form: a rod of non-magnetic "Bakelite," $486 \mathrm{~cm}$. in length and $2.4 \mathrm{~cm}$. in diameter, was turned down in a lathe so as to form a spool at one end, whose length was $50 \mathrm{~cm}$. and diameter $1.4 \mathrm{~cm}$. (see Fig. 2). At the middle of this long spool another channel was cut out $2 \mathrm{~cm}$. long and

${ }^{3}$ Phil. Trans. Roy. Soc., p. 432, vol. 198, Ig02.

- Specimens of hard rubber showed magnetic properties. 
$2 \mathrm{~mm}$. deep. In this smaller channel 600 turns of No. 37 silkcovered copper wire was wound and the ends carried along in a groove to two binding posts at the end of the "Bakelite" rod. This secondary or exploring coil was covered with hard wax and smoothed to the same diameter as the spindle, which was $50 \mathrm{~cm}$. long, on which the primary was to be wound. The rod was cleaned as thoroughly as possible to free the surface from any particles of steel which might have stuck in turning down the rod in the lathe. For the primary, No. I 8 double cottoncovered copper wire was used and wound in four layers, which just flled the channel turned out for the primary. As each layer was put on it was thoroughly shellacked. The winding was done on a lathe and all parts of the wire and coil were kept from contact with the iron parts of the lathe. The screw-cutting attachment was adjusted so that the wire, as it was fed onto

FIG. 2.

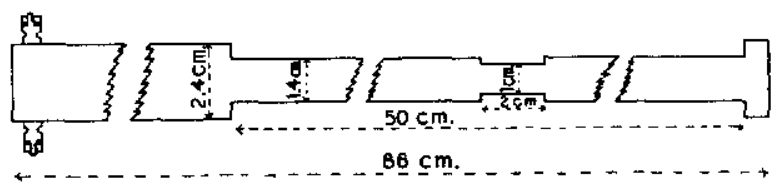

the coil, was carried along at the same rate as the windings of the coil. While the number of turns for each layer was not the same, yet this arrangement for feeding the wire to the coil gave the number of turns per centimetre in each layer with good uniformity. There is no question but what the method employed by Jenkins ${ }^{5}$ and others in winding solenoids gives greater uniformity in the number per unit length than the method employed in this work. Jenkins accomplished this end by cutting an accurate thread along an insulating tube and winding bare wire under tension in this groove. Inasmuch as I wanted a standard coil of several layers, this seemed impractical unless the coil could have been built out of a series of telescoping "Bakelite" tubes. The small inner diameter of the solenoid to be tested prevented the use of more than two tubes.

The constant of the standard coil was computed by means of the well-known formula ${ }^{6}$ already given for solenoids. The

'Jenkins, Phil. Mag., Ser. 6, vol. 26, p. 752, 1913.

"Watson, "A Textbook of Practical Physics," p. 522, I906. 
total field strength was taken as the sum of the fields due to the various layers. For a long time there was a persistent difference between the computed and observed values of the solenoid tested. In the case of the solenoid, one metre in length, this difference amounted to about one-half of one per cent. and was always in a direction such that the computed value was less than the experimental. After investigating, seemingly, every possible chance of error, I finally found, what apparently has been overlooked in many important researches, that in the various coils which were tested, $n$, the number of turns per unit length, was invariably greater at the centre than at the ends. This is, I believe, due to the fact that as the wire comes from one layer up to the next one at each end of the coil in the wind-

Fig. 3.

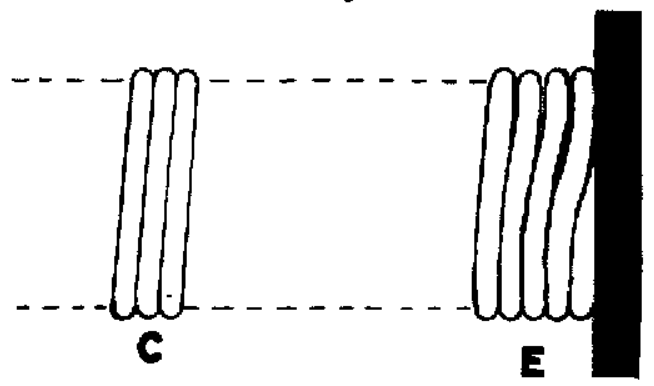

ing, the turns which lie next to the wire where it comes up must curve out around it, and in so doing cannot be made to lie so close together as at the middle of the coil where the turns have straightened out (see Fig. 3). Ordinarily we divide the total number of turns in a layer by the length of the layer in order to find $n$. This leads to a systematic error, as indicated above, if, as I have found, the value of $n$ is smaller at the ends than in the middle of the coil. ${ }^{7}$ The turns at the ends do not have so much effect on the field at the centre as those turns wound about the centre, and therefore they should not be given equal weight with those at the centre when it comes to finding the value of $n$. The correction for this discrepancy was made by finding $n$ for various parts of the coil and then the average when these various values of $n$ had been weighted proportional to their

"This effect is very noticeable in the windings of the field coils on older types of dynamos; in fact. it must be present in every solenoid as ordinarily wound. 
effects upon the field at the centre of the coil. When this was done the computed and observed values of the field at the centre of the coil agreed within the limits of accuracy of the method, which was I : Iooo. The fact that the value of $n$ was less at the ends of the coil than at the centre may be shown in another way. If the observed values of the field intensity along the axis of the coil are compared with the computed it will be found that the observed values drop off more rapidly than the computed as one passes from the centre out toward the ends. This is shown in Fig. 4.

The large solenoid to be tested, of which mention has already

FIG. 4 .

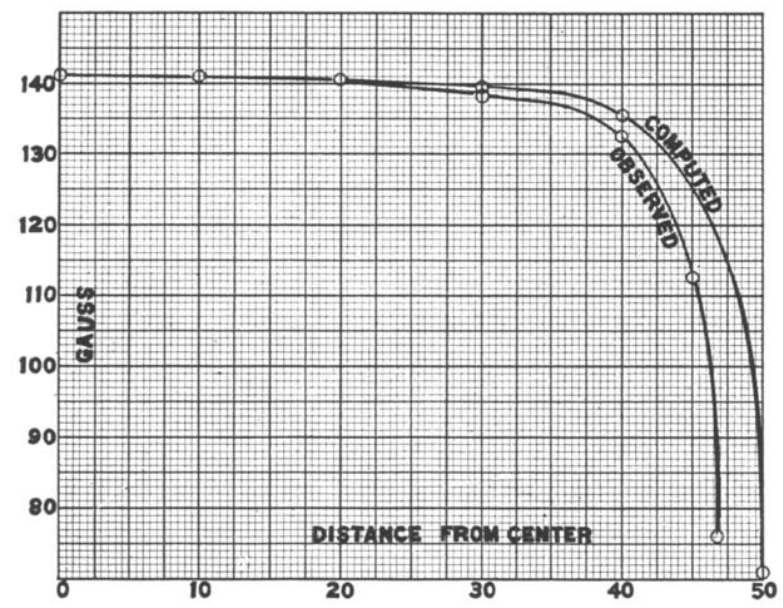

been made, was wound in 24 layers and had a total of $I I, 25 I$ turns. It was wound on a brass tube, $2.54 \mathrm{~cm}$. inner diameter and $3.8 \mathrm{~cm}$. outer diameter. This tube, with its end pieces, was slotted lengthwise to prevent induction currents when alternating currents were sent through the coil. The computed constant was obtained in the manner just indicated and found to be I42.362 gauss, while the value as determined experimentally was I42.445. The last value was the average of twenty observations. This difference means a discrepancy of less than one part in a thousand between the observed and computed values, which for this particular coil means that not more than Io to 15 
turns could be cut out by a short circuit without being able to detect it. However, by refinements which have been indicated, such as methods of winding and construction, greater accuracy can be secured, and there seems to be no reason why the accuracy could not be pushed to one part in 10,000 (I: I0,000). It would seem that in the end the accuracy of the method rested upon what refinement could be made in detecting changes in the current which flowed in the two coils. The best instrument which I had could be read only to one part in one thousand ( $I: I 000$ ). It is to be noted that in this method it is not a matter of reading the absolute values, but merely detecting variations.

The ballistic galvanometer was a Leeds \& Northrup instrument, type $\mathrm{HB}$, having a resistance of $18 \mathrm{ohms}$, a period of deflection equal to 9 seconds, and whose sensibility is 122 megs. It seemed to be a very satisfactory instrument for this kind of work.

The standard coil was next used on a solenoid ${ }^{8}$ which had seen service in previous work and also on an ordinary tangent galvanometer coil and a Helmholtz coil, all of whose constants could be computed with a fair degree of accuracy. Thus not only were the constants of the various coils ascertained with greater certainty, but, conversely, the constant of the standard coil could be said to have been more firmly established.

\section{SUXIMARY.}

I. This paper has given a practical method for testing the constant of a solenoid by experimental means.

2. The method recommends itself because of its simplicity. The constant of the solenoid is determined in terms of the deflections of a ballistic galvanometer and the constant of another coil taken as a standard. If a long, slim solenoid is used for the standard, the only quantity which must be known with great accuracy in determining its constant is the number of turns per unit length. The constant of the ballistic galvanometer does not need to be known, neither does the ammeter, which indicates the constancy of the current in the coils, need to be calibrated. Even the exploring coil does not need to have its inductive area determined. Once the constant of the standard coil has been definitely fixed, the method could not be much simpler.

Bhys. Rev., loc, rit. 
3. It is evident that the exploring coil could have been placed first in one coil and then in the other and the deflection observed when only one field was suddenly reduced to zero. In such a case,

$$
S=G \stackrel{d_{2}}{d_{1}}
$$

This last equation appears simpler. The author believes, however, that by keeping the two coils in series it will be easier to keep the current constant, because there is absolutely no change to be made in the circuits except to throw a reversing switch for one coil. Using the coils separately would necessitate change of circuits; also keeping the coils in series insures that in breaking the circuit the field due to both coils must vary simultaneously. Of course, difference in rate of decay of the magnetic fields should not affect the results if the period of deflection of the galvanometer is large enough.

4. An investigation as to what ratio of $S / G$ would give the least error in $S$ shows that when $S / G=$ I the error in $S$ should be a minimum. This is an impossible condition, as the $d_{1}$ in equation (2) becomes equal to zero. If the experiment is worked as a zero method it would be necessary to have each coil on a separate circuit and so arranged that the two fields could be made to just neutralize each other. When this occurred there would be no deflection of the galvanometer and equation (2) would then be written:

$$
\begin{gathered}
I_{1} S-I_{5} G=0 \\
S=G \frac{I_{2}}{I_{1}}
\end{gathered}
$$

This was tested out, but was not so sensitive as the method finally pursued. In this zero method it would be necessary to know the absolute value of the current, which would mean the calibration of one or two ammeters.

5. This work has shown that great care must be used in winding a solenoid if the constant is to be obtained by computation. The author would recommend concentric "Bakelite" tubes where several layers are to be employed, and the winding carried out similar to the method used by Jenkins. ${ }^{9}$ If the constant of the solenoid is to be determined by the method sug-

'Jenkins, loc. cit. 
gested in this paper, then the winding need not be carried out with such extreme care.

6. Investigating the constant of a solenoid has brought out the fact with renewed earnestness that what we work with in a great many experiments is not a uniform field all along the axis of the solenoid, but, as shown by Fig. 4 , it is a field that is continually decreasing as one goes from the centre of the coil out toward the ends. In work of measuring the magnetic properties of various substances this is too frequently lost sight of, and in studying such phenomena as the magnetostrictive effects everyone seems to have fallen into the error of considering the value of the field strength at the centre of the solenoid as holding constant very nearly to the ends. A consideration of the equation for the field strength at any point along the axis of a solenoid will soon convince one of this point, viz.,

$$
H=2 \pi n 1\left[\frac{D+\frac{l}{2}}{\sqrt{\left(D+\frac{l}{2}\right)^{2}+R^{2}}}-\frac{D-\frac{l}{2}}{\sqrt{\left(D-\frac{l}{2}\right)^{2}+R^{2}}}\right]
$$

where $D$ is the distance from the centre of a solenoid of length $l$ and radius $R$. It was from this equation that the computed values for Fig. 4 were obtained.

So far as the author knows no one has done in a practical way for solenoids what Maxwell did for galvanometer coils, viz., determine what the form of a coil should be in order to get certain desired fields. At present work is being done on this problem. It seems quite certain that the field may be kept up to a constant value along the axis much farther from the centre than is now done by ordinary solenoids

In closing, expressions of appreciation are due Mr. Ray Calhoon, an advanced student in the department, for the valuable assistance rendered in making long and tedious series of observations.

Physical Laboratory, Oberlin College, Oberlin, Ohio. 